\title{
Vitrectomy, argon laser, and gas tamponade for serous retinal detachment associated with an optic disc pit: a case report
}

\author{
M P Snead, N James, P M Jacobs
}

\begin{abstract}
We report the case of a 9-year-old boy with a right optic disc pit and associated serous retinal detachment of the macula. This was treated by vitrectomy, endolaser, and gas tamponade as a primary procedure, which resulted in complete resolution. We review the literature and conclude that further reports of the management of this uncommon condition are required so that firmer guidelines on treatment can be established.
\end{abstract}

A 9-year-old Caucasian boy presented to the eye casualty department in July 1989 with a sixmonth history of decreased visual acuity. A year previously an optician had recorded unaided visual acuities of $6 / 6$ right eye and 6/6 left eye. There was no relevant past ocular or medical history of note.

On examination his unaided visual acuities were 6/6 left and 1/60 right eye. There was no improvement with refraction. The anterior segment appeared normal, he had a right relative afferent pupil defect, and funduscopy revealed a serous retinal detachment of the macula clearly linked to an optic disc pit in the right eye. The detached retina had undergone cystic retinal degeneration, and the underlying pigment epithelium was irregularly pigmented (Fig 1). The left fundus was normal. The patient thought that there had been a recent improvement in the vision of the right eye, and treatment was therefore deferred and serial observations carried out. After nine months no further improvement had occurred. Various methods of treatment (see 'Discussion') were considered, and, though laser photocoagulation alone has met with some success, in view of the patient's age this would

University Hospital, Nottingham M P Snead

N James

P M Jacobs

Correspondence to:

M P Snead, FRCS

Department of

Ophthalmology, University

Hospital, Nottingham NG7 2UH.

Accepted for publication 13 November 1990

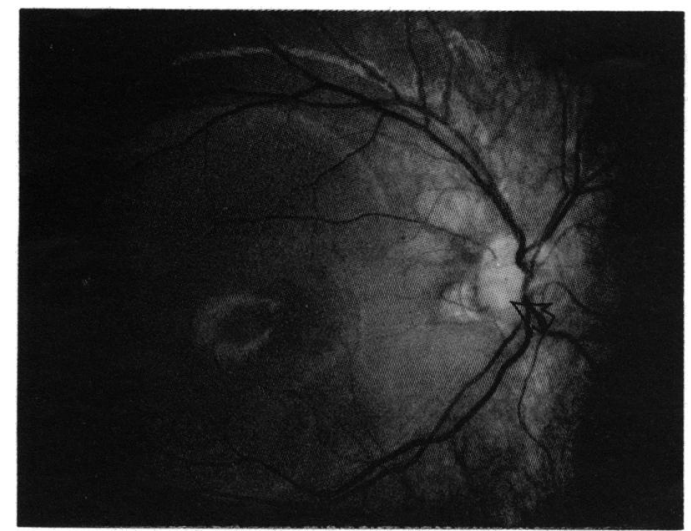

Figure 1 Preoperative fundus photograph. Arrow indicates optic disc pit.

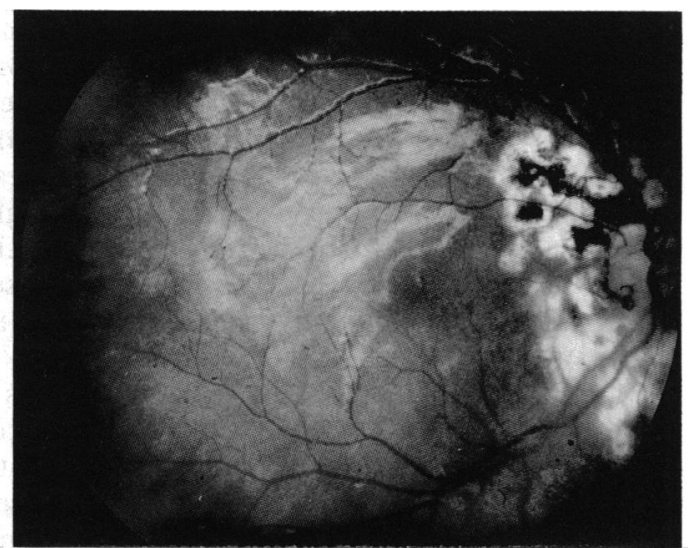

Figure 2 Postoperative fundus photograph.

have required a general anaesthetic. Vitrectomy and laser photocoagulation followed by fluid/gas exchange seemed to offer the highest chance of success with a single procedure (and anaesthetic), and it was agreed to proceed with surgery.

On 10 April 1990 the patient underwent right pars plana vitrectomy, endolaser treatment, and $40 \%$ SF6 exchange. A standard $20 \mathrm{~g}$ three-port pars plana vitrectomy was performed, and during surgery the vitreous was noted to be still attached. Argon endolaser was applied temporal to the disc in a confluent double line of burns to seal off the link between pit and detachment and extended superiorly and inferiorly into attached retina. This was applied after the vitrectomy and before fluid-gas exchange in order to minimise any laser induced damage to the papillomacular bundle. A power setting was used which was just sufficient to produce a soft white burn in an area of attached retina and then applied in the distribution described above. This produced minimal or no reaction in the pigment epithelium underlying the detached retina at the time of application. There was no evident communication between the detachment and the vitreous cavity, and no attempt was made to drain subretinal fluid. The posterior segment was filled with $40 \%$ SF 6 in air.

Postoperatively the posterior retina was attached, but there was a collection of subretinal fluid inferiorly. It appeared that the previously submacular fluid had been displaced inferiorly by the gas bubble. This fluid was slowly absorbed over 16 weeks. At five months the retina was completely attached and the visual acuity had improved to $6 / 60$ (Fig 2).

\section{Discussion}

An association between optic disc pit and macular 
detachment is well recognised. Sugar ${ }^{1}$ notes that macular lesions have been described in 51 of 157 cases of optic disc pit. Gass ${ }^{2}$ in a study of five case reports suggests that serous detachment of the retina extending from the optic disc pit into the macular area is the basic lesion and that cystic retinal degeneration, macular hole formation, and retinal pigment epithelial disturbances are all secondary to the fluid which gains entrance to the subretinal space. Similar findings with the morning glory disc abnormality have led to suggestions that these two conditions are merely variations of the same basic abnormality. ${ }^{3}$

The origin of the subretinal fluid has aroused further interest, and the vitreous, subarachnoid space, inflammatory processes, abnormal blood vessels and orbital tissues have all been suggested as a source..$^{3-6}$ Although internal traction has been implied as a mechanism associated with these detachments in several reports, there is only a single case report recording a retinal break as a contributing factor. ${ }^{7}$

The varied opinions on the origin of the subretinal fluid and consequent mechanisms underlying the associated retinal detachment have led to a wide range of reports on different approaches to management. In Gass's series of five patients ${ }^{2}$ one underwent spontaneous resolution over 18 months without treatment. In the other four the macula remained detached despite three cases having treatment with either photocoagulation 'barrage' temporal to the pit or attempted alteration of the intraocular pressure. The results led Gass to state that either photocoagulation followed immediately by drainage of subretinal fluid or surgical decompression of the dural sheath of the optic nerve may prove to be more successful. Irvine $e t a l^{3}$ describe such a case of optic nerve sheath fenestration in a previously vitrectomised eye with morning glory disc and macular detachment, observing gas from the fluid-gas exchange bubbling through the nerve sheath fenestration, confirming the communication between optic nerve and vitreous cavity.

In a study of 18 patients Abujamra et al $l^{8}$ treated 10 with photocoagulation and achieved full reattachment in only one case. Kapel $e t a l^{9}$ reported on 14 patients with optic disc pit, eight of whom had an associated macular detachment and five were treated with argon laser, all unsuccessfully, and they concluded that no effective treatment was available for this serious complication.

There have been some more encouraging results. Kottow ${ }^{10}$ treated two patients with laser photocoagulation with successful reattachment in both, though no information is given beyond two months and seven days respectively. Annesley $e t a^{11}$ published a series of five cases of optic disc pit with macular detachment. In four cases successful reattachment was achieved with krypton laser alone, but three required more than one treatment session. The remaining patient underwent successful reattachment with krypton laser, vitrectomy, and fluid-gas exchange following two unsuccessful treatments with laser alone. Schatz and McDonald ${ }^{12}$ treated five of six cases of optic disc pit with associated retinal detachment, two with laser photocoagulation alone and three combined with pars plana vitrectomy and fluid-gas exchange, all with eventual retinal reattachment. Von Fricken $e t$ $a l^{7}$ describe successful reattachment following vitrectomy and fluid-gas exchange in an unusual case of morning glory disc with rhegmatogenous retinal detachment.

This case report documents the successful reattachment of a serous macular detachment associated with optic disc pit by vitrectomy, endolaser, and fluid-gas exchange. The limited recovery in visual function is probably related to its longstanding nature and to the deposition of exudate on the posterior retinal surface and marked alteration in macular function characteristic of these detachments. ${ }^{2}$ Further reports on the treatment of this uncommon condition are required so that clearer guidelines on management may be defined.

1 Sugar HS. Congenital pits in the optic disc and their equivalents (congenital colobomas and coloboma-like excavations) associated with submacular fluid. Am F Ophthalmol 1967; associated with $298-307$.

2 Gass JDM. Serous detachment of the macular secondary to congenital pit of the optic nerve head. Am $\mathcal{F}$ Ophthalmol $1969 ; 67: 821-41$

3 Irvine AR, Brookes Crawford J, Sullivan J. The pathogenesis of retinal detachment with the morning glory disc and optic pit. Retina 1986; 6: 146-50.

4 Kindler P. Morning glory syndrome: unusual optic disc anomaly. Am $\mathcal{F}$ Ophthalmol 1970; 69: 376-84.

5 Hamada S, Ellsworth RM. Congenital retinal detachment and optic disk anomaly. Am $\mathcal{F}$ Ophthalmol 1971; 71:460-4.

6 Adelung K, Aulhorn E, Thiel H-J. Functional disturbances associated with pitting of the optic disc. Klin Monatsbl Augenheilkd 1987; 191: 1-7.

7 von Fricken $M$, Dhungel $R$. Retinal detachment in the morning glory syndrome-pathogenesis and management. Retina 1984; 4: 97-9.

8 Abujamra S, De Souza EC, Da Cunha SL. Pitting of the optic disc. Rev Bras Oftalmol 1984; 43: 169-73.

9 Kapel J, Otradovel J, Dotrelova D. Maculopathies associated with congenital pits of the optic disc. Czesk Oftalmol 1983; 39: 9-17.

10 Kottow M. Photocoagulation of optic disc pits. Ophthalmologica 1982; 184: 26-9.

11 Annesley W, Brown G, Bolling J, Goldberg R, Fischer D. Treatment of retinal detachment with congenital optic pit by krypton laser photocoagulation. Graefes Arch Clin Exp krypton laser photocoagulation

12 Schatz H, McDonald $\mathbf{R}$. Treatment of retinal detachment associated with optic nerve pit or coloboma. Ophthalmology 1988; 95: 178-86. 